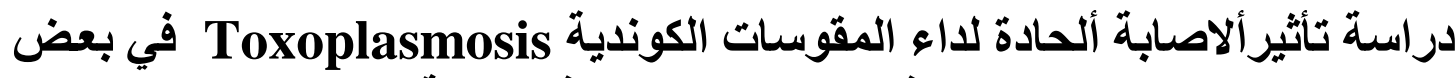

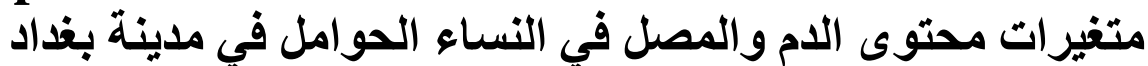

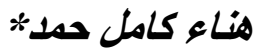

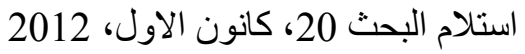

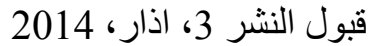

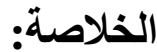

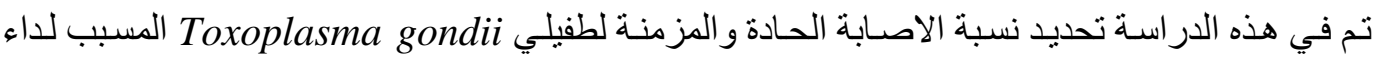

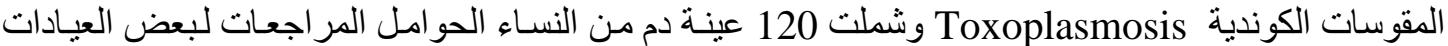

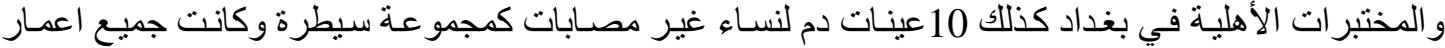

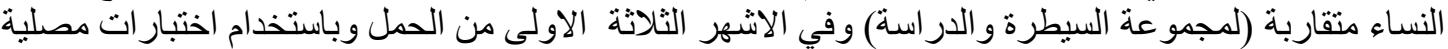

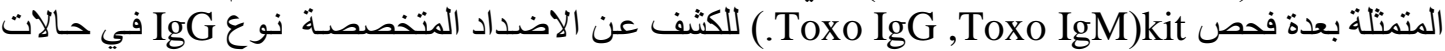
الاصابة المزمنة والاضداد المتخصصة نوع IgM في الحالات الحادة بطريقة Electro Clia باستخدام جهاز

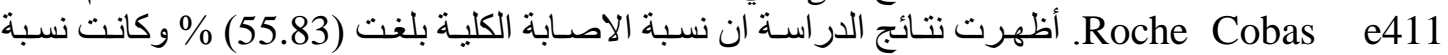

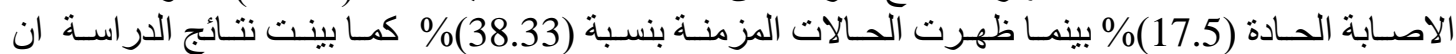
الاصعابة الحادة بطفيلي المقوسات الكوندية

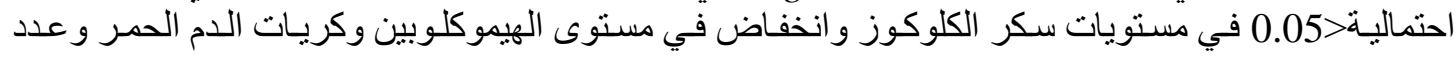
الصفيحات الدموية وارتفاع عدد الكريات البيض عند مقارنتها بمجمو عة السبطرة .
\end{abstract}

الكلمات المفتاحية : المقوسات الكوندية ؛ النساء الحوامل؛ Electro clia ؛ سكر الكلوكوز

Oocysts

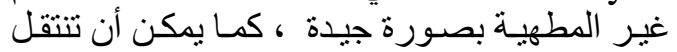

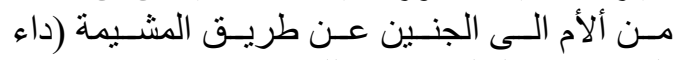

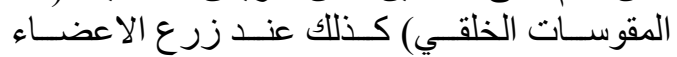

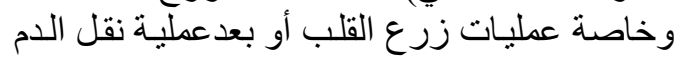

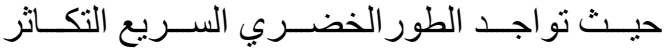

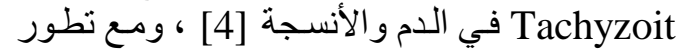

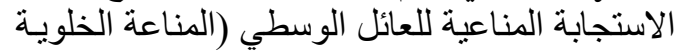

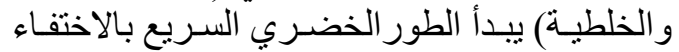

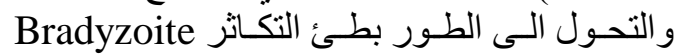

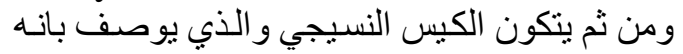

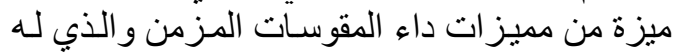

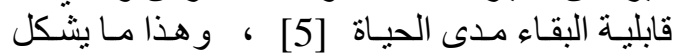

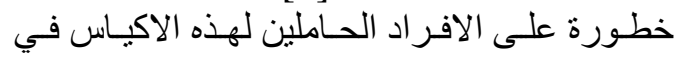

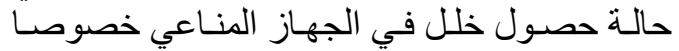

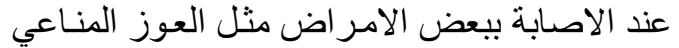

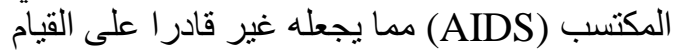
بوظائفه او بتأثثر عوامل اخرى مثل العمر والحالة

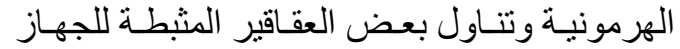

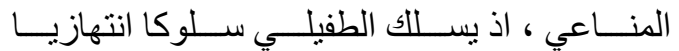
Opportunistic

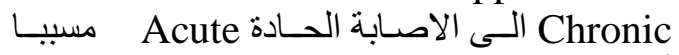

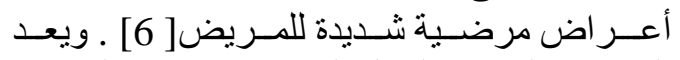

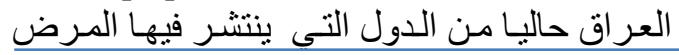

المقدمة:

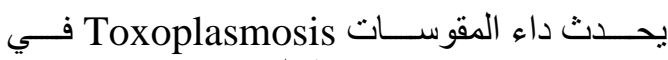
Toxoplasma الإنسـان نتيجـة الإصـابة بطفيلـي gondii

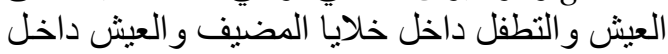

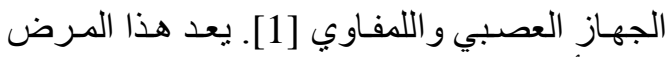
مـن الأمسر اض المشـتركة zoonose بـين الإنســان

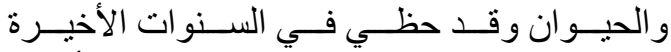
باهتمامـات طبيـة نظر ال النتشـاره الو اسـع في أنحـاء

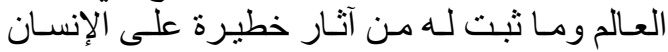

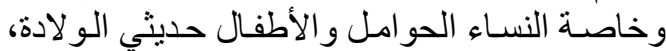

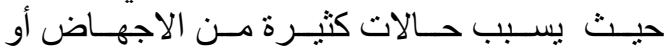

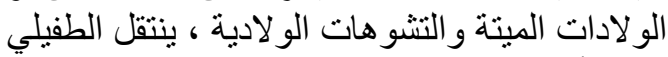

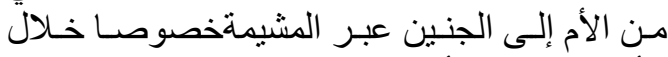

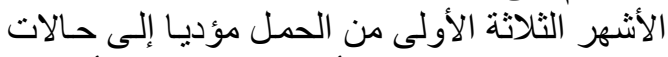

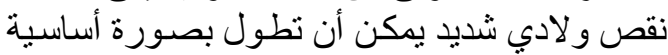

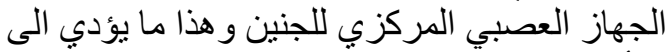

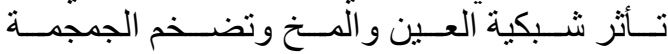
بالسو ائل (استسقاء الدماغ) Hydrocephaly وقد الند

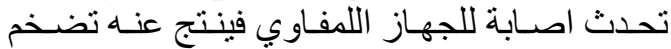
الكبـد و الطحسال hepatospleenomegaly

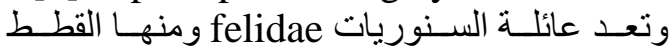

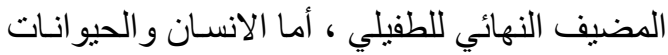

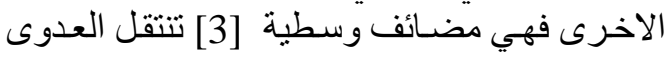

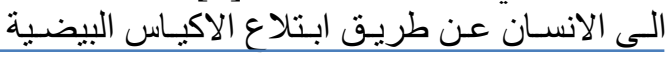

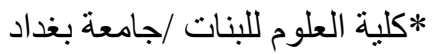


Roche نوع IgM في المصول البشرية في حالات الاصنابة

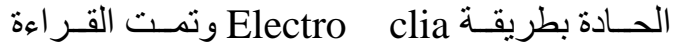

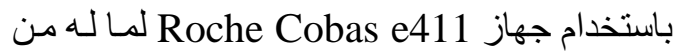
خصوصية وحساسية عالية في التفريق بين الأصابة الحادة و المزمنة.

2- تعيين مسنوى سكر الكلوكوز في مصل الدم استخدمت طريقة Zweig and Campbell

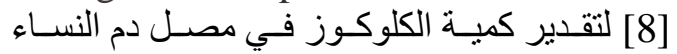

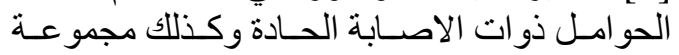

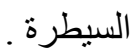

3- اختبار ات الدم Haemotology Tests أعتمدت طريقة Voigt [9 [9 لتقدير مستوى

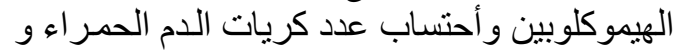

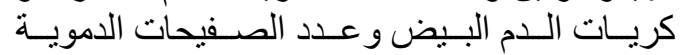
Platelets

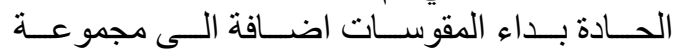

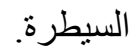

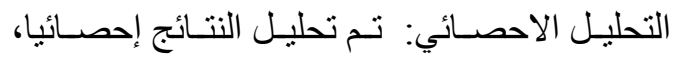
و أعرب عن قيمها (mean I SED) و تم تحديد الاحي مستوى الدلالة باستخدام اختبار (t).

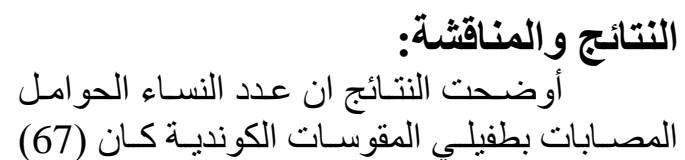

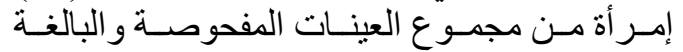

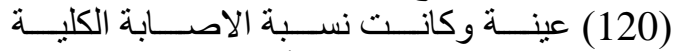

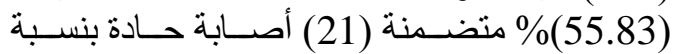

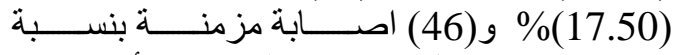

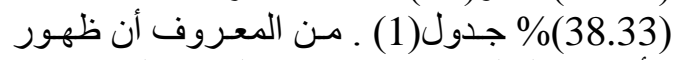

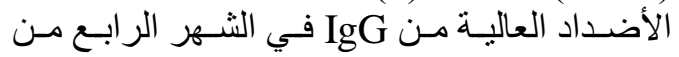

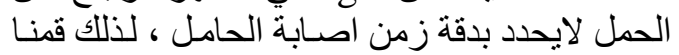

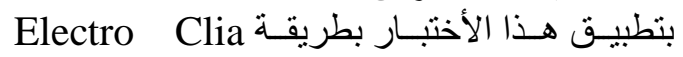

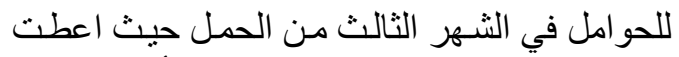

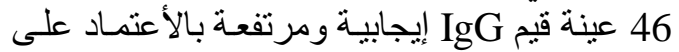
الألفة الوظيفية لأضداد IgG النو عية للمقوسـات بعد الأنداد

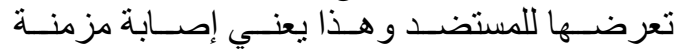

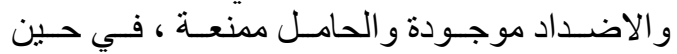

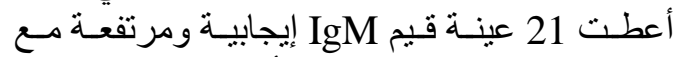

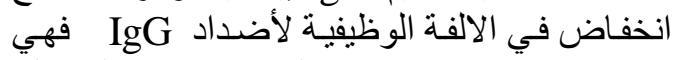

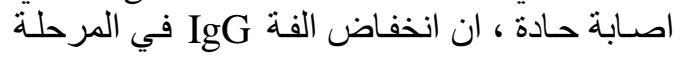

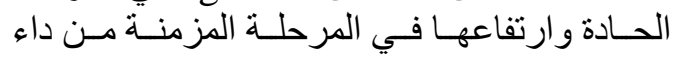

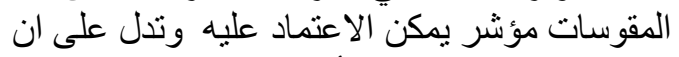

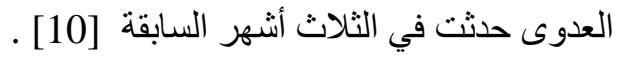

مـن حيـث ارتفــاع اعـداد النســاء الحوامـل اللاتـي

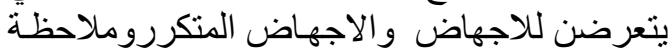

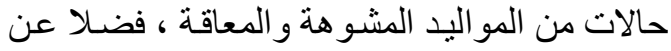

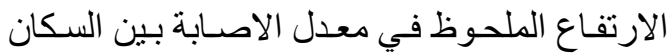

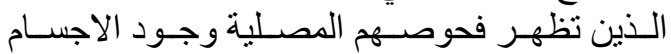

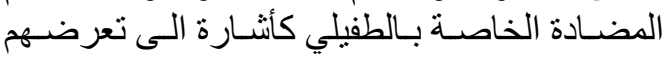

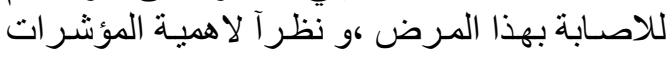

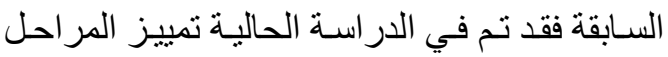

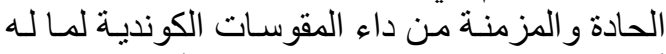

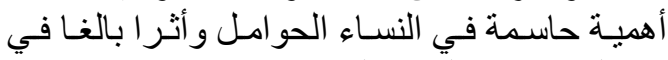

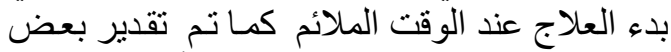

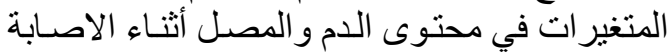

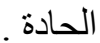

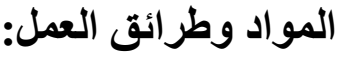

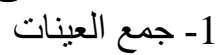

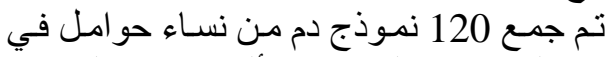

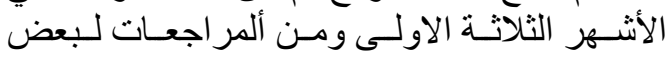

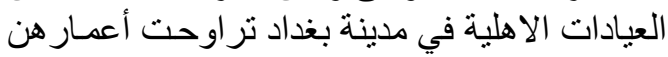

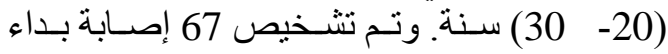

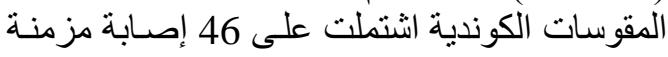

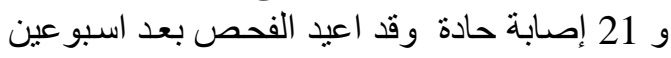

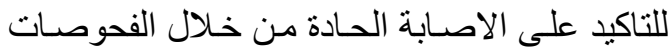

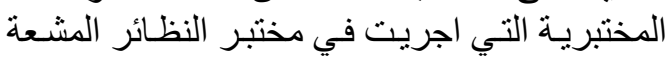

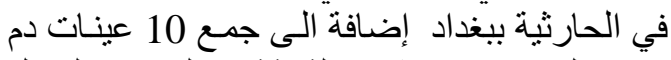

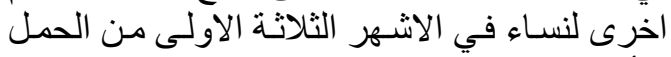

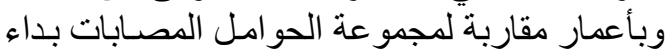

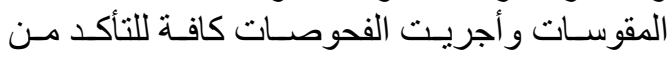

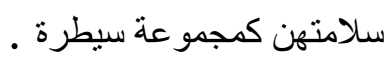

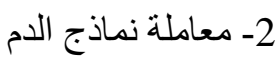

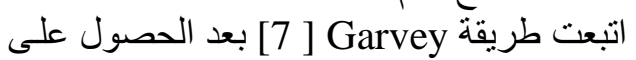

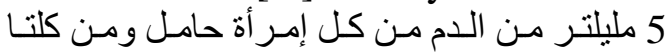

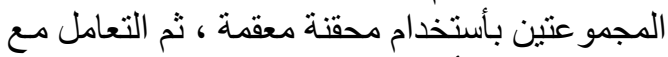
كل نموذج كما يأتي : كلين

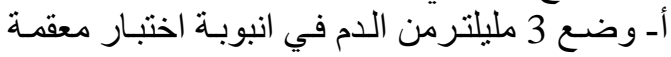

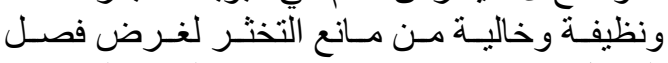

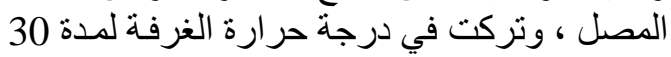

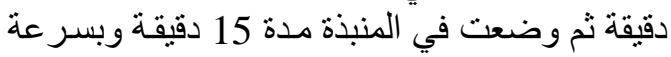
2000 دورة / الدقيقة .

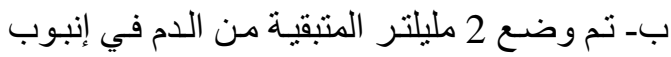

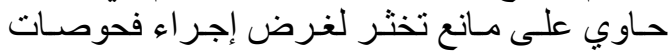

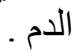

Serological tests الاختبارات المصلية

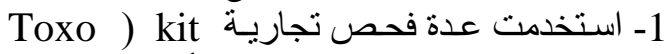
(IgG

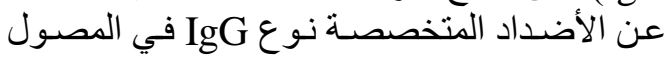

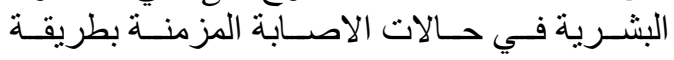
Electro Clia تجاريـة Toxo IgM) kit مـن انتـاج شـركة 


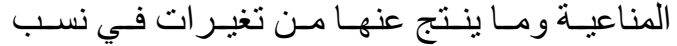

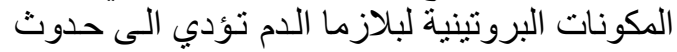

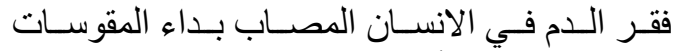

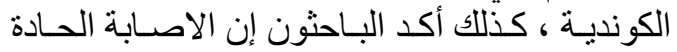

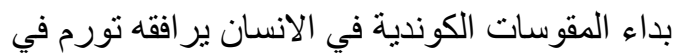

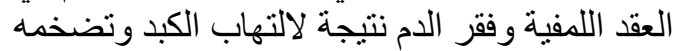

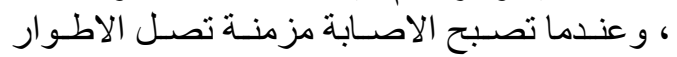

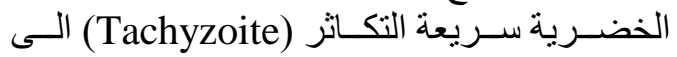
الأماغ و القلب و العضـلات الهيكلية ويكون تكاثر هـا

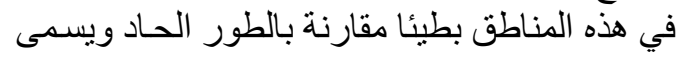

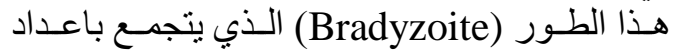

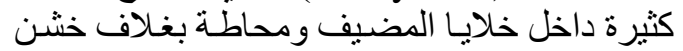

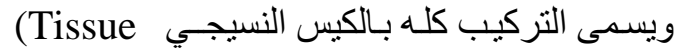
الى ع.cyst)

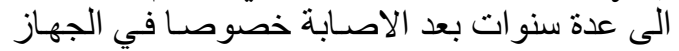

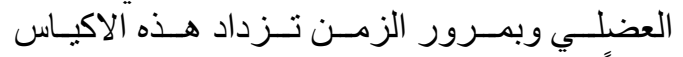

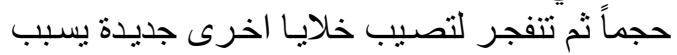

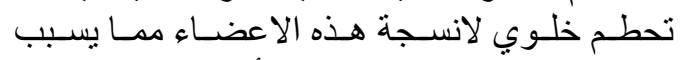
حدوث بقع نزفية شديدة و هذا ما أكده [15].

\section{جدول (3): المتغيرات في معدل ومستوى بعض الفضو الفي

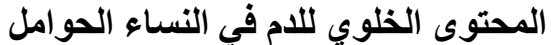

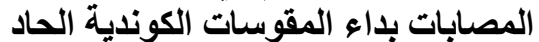

\begin{tabular}{|c|c|c|c|}
\hline $\begin{array}{c}\text { Hematological } \\
\text { Parametr }\end{array}$ & $\begin{array}{c}\text { Acut infaction } \\
(\mathrm{n}=21)\end{array}$ & $\begin{array}{c}\text { Control } \\
(\mathrm{n}=10)\end{array}$ & $\begin{array}{c}\text { P- } \\
\text { Value }\end{array}$ \\
\hline $\begin{array}{c}\text { count(cells/ul) } \\
\text { WBC }\end{array}$ & $1,567,16 \pm 546$ & $\begin{array}{c}\text { Mean } \pm \text { SD } \\
\text { Mean } \pm \text { SD }\end{array}$ & $P<0.05$ \\
\hline $\begin{array}{c}\text { RBC count } \\
\text { (cells/ul) }\end{array}$ & $\begin{array}{c}1,333,067 \pm 282,3 \\
79\end{array}$ & $\begin{array}{c}217,260 \pm 36.2 \\
12\end{array}$ & $\begin{array}{c}P< \\
0.05\end{array}$ \\
\hline $\begin{array}{c}\text { platelet count } \\
\text { (cells/ul) }\end{array}$ & $\begin{array}{c}76,899,15 \pm 5,244 . \\
91\end{array}$ & $\begin{array}{c}12.66 \pm 5511.9 \\
5\end{array}$ & $\begin{array}{c}P< \\
0.05\end{array}$ \\
\hline $\begin{array}{c}\text { Hemoglobin(g/ } \\
\text { dl) }\end{array}$ & $3.84 \pm 0.67$ & $0.63 \pm 0.11$ & $\begin{array}{c}\mathrm{P}<0.00 \\
1\end{array}$ \\
\hline
\end{tabular}

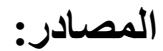

1-Assmar, $\quad$ M.; $\quad$ M.H.Manili; A.E.Rastaghi and S.N.Dezfooli1999. Immunogenicity of gammairratiated.Toxoplasma gondii tachyzoites in mice.Iran Biomed .J.3(34):93-97

2-Dubey, J.P.

2002: Toxoplasma gondii: transmission, diagnosis and prevention. Clin Microbiol. Infect., 8:634-640.

3-John,D.T.and Ptetri, W.A.2006 Marrkeil and Voges Medical Parasitology, $9^{\text {th }} \quad$ Edit.W.B.Saunders Company.

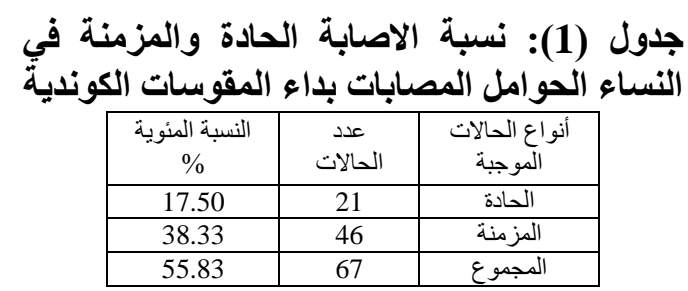

جدول (2): التغيرات في معدل مستوى سكر الكلوكوز في مصل دم النساء الحوليرات فئل الدول المصابات بداء المقوستات الحاد ومجموعة السيطرة

\begin{tabular}{|c|c|}
\hline 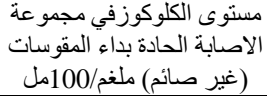 & 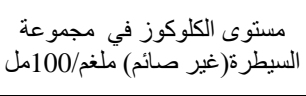 \\
\hline 71.3 & 112.2 \\
\hline
\end{tabular}

يظهر في الجدول (2) ان مستوى الكلوكوز في دم

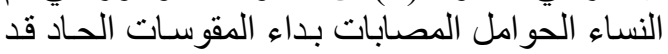

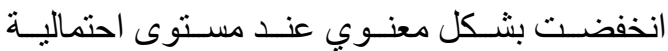

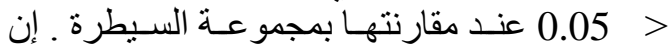

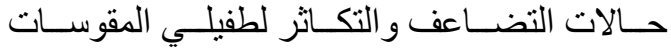

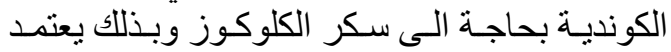

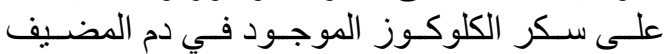

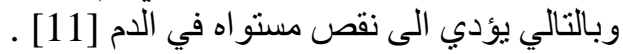

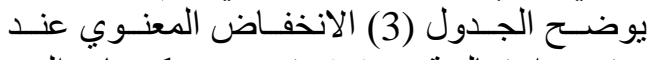

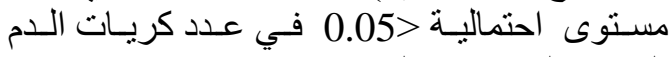
الحمر و الصفيحات الدموية و> > 0.001 في مستوى فئوى

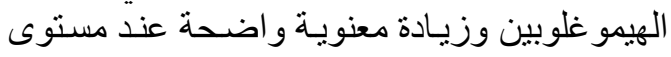

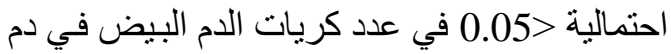

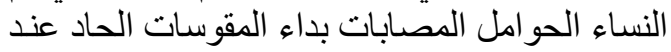

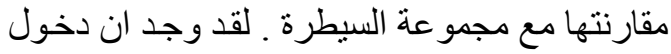

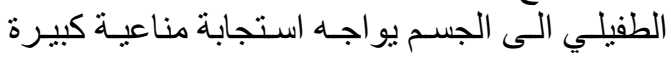

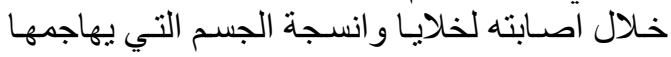

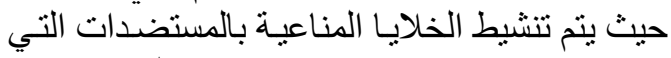

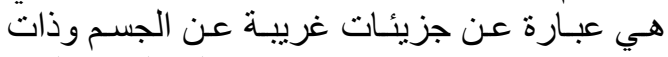

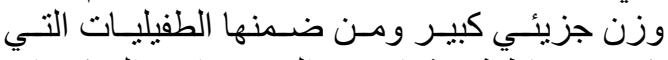

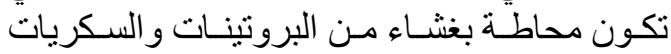

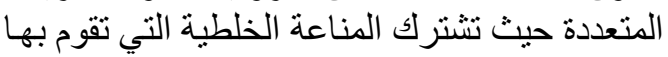

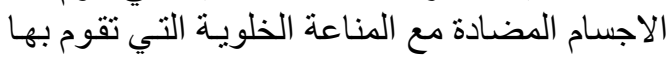

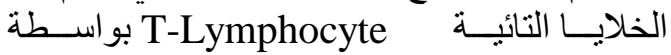

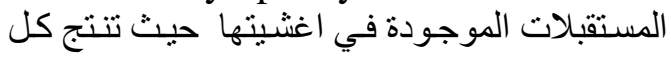

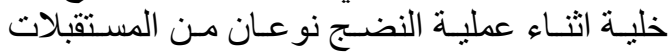

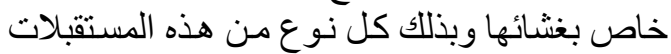

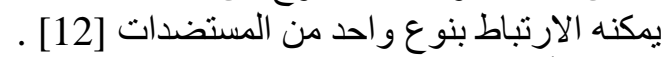

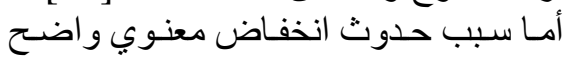
في عدد كريات الدم الحمرومستوى الهيمو غلوبنوبين

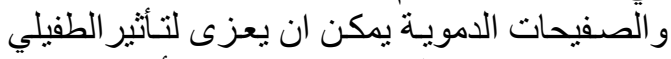

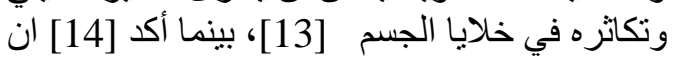

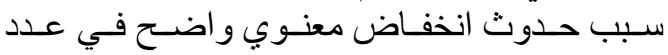

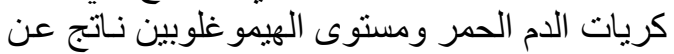

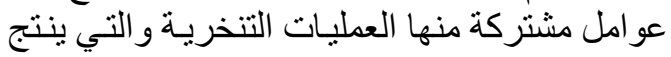

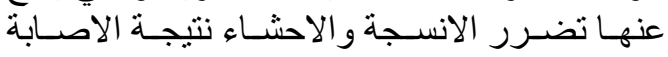

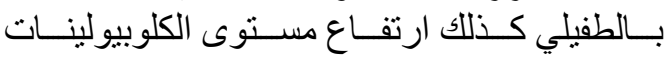


10-Flori, P.;Bellete, B.; Crampe, C.and Maudry,A.2008. Technique for dating toxoplasmosis in pregnancy and comparison with the Vidas antiToxoplasma $\mathrm{IgG}$ avidity test. Clin.Microbiol.Infect., 14:242-249.

11-غسان فتحي محمد. ( ـ ـ تأثير داء المقوسـات

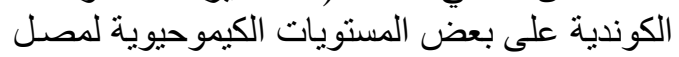

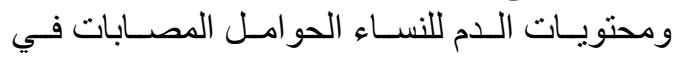
الموصل مجلة التربية و العلم ـ المجلد (24 ) العدد 4 صفحة (55-50).

12-Pier, G., Ceri, H.;Mody. C.and Preston, M. 2004. T cell Maturation and Activation. In: Immunology, Infection and Immunity Pier, G.B., Lyczak, J.B. and Wetzler, L. M. eds, ASM Press, Washington, DC. pp. 315342.

13-Jonies, T. C.; Hunt, R. D.and King, N. W. 1997. Veterinary Pathology. 6th ed. Lippin Cott Williams \& Wilkins, Awolters Kluwer Company, USA,: 555- 560.

14-Advincula, J.K.C.and Lewide, S.Y.P.2010.Cabanacan- Salibay CSerologic detection of Toxoplasma gondii. Sci. Med. 2010; 20(1):76-82.

15-Montoya, J.G. 2002.Laboratory diagnosis of Toxoplasma gondii infection \&Toxoplasmosis. Infect. Dis.185(1):73-82.
4-Yamamoto, J. H. ; Filho , J. K. and Gazzinelli, $\mathrm{R}$. T. 2000.Discrimination between patients with acquired toxoplasmosis and congenital toxoplasmosis on the basis of the immune response to parasite antigens. The Journal of infectious diseases, 181(6):2018-2022.

5-Wilson, M. Mc.and Auley, J.M.1999.Toxoplasma. In: Murray PR, ed. Manual of clinical microbiology. 7th ed.Washington,D.C.:American Society for Microbiology :13741382.

6-Remington, J.S.; Mc Leod, R.; Thulliez, P.and Desmonts, G. 2001. Toxoplasmosis. In: Remington J.S.; Klein, JO. eds. Infectious diseases of the fetus and newborn infant.

7-Garvey, J. S.; Cremer, N.E. and Sussdorf, D.H. 1981. Methods in Immunology. 3 rd. ed.W. AQ Benjamin, Inc.: 297.

8- Zweig, M. H. and Campbell, G. 1993. A fundamental evaluation tool in clinical medicine. Clinical Chemistry, 39 . (4): 561-577.

9-Voigt, G. L. 2000. Hematology Techniques Concepts for Veterinary Technician, 1st ed., lowa state university press: $28-52$. 


\title{
Effect Study the of Acute toxoplasmosis on Some Blood and Serum Parameters in Pregnant Women in Baghdad City
}

\begin{abstract}
Hanaa K. Hamad *
*University of Baghdad / College of Science for Women

Abstract:

In this study the rate of infection in acute and chronic Toxoplasma gondii parasite that causes toxoplasmosis was determined. This study was Included 120 blood samples that collected from pregnant women revisions to some clinics and laboratories in Baghdad civil as well as 10 blood samples from non-infected women as a control group. All blood samples were collected in the first three months of the pregnancy period for detection toxoplasmosis by using serological tests of test kit ( Toxo , IgG.,Toxo , IgM ). To detect antibodies specialized type of IgG \&IgM in acute and the chronic infection by Electro Clia manner using a Roche Cobas e411. The results showed that the total infection rate was $(55.83) \%$, the rate of infection in acute (17.5)\% While the rate of chronic infection was (38.33)\%. As the results of the study showed that rate of acute infection of toxoplasmosis in pregnant women was significant decrease occurs when the level of probability of $<0.05$ in glucose sugar levels, and a decrease in the level of hemoglobin and red blood cells, platelets and the high number of white blood cells when compared with the control group .
\end{abstract}

